\title{
Partial discharges in ellipsoidal and spheroidal voids
}

\author{
Crichton, George C; Karlsson, P. W.; Pedersen, Aage
}

Published in:

IEEE Transactions on Electrical Insulation

Link to article, DOI:

10.1109/14.90292

Publication date:

1989

Document Version

Publisher's PDF, also known as Version of record

Link back to DTU Orbit

Citation (APA):

Crichton, G. C., Karlsson, P. W., \& Pedersen, A. (1989). Partial discharges in ellipsoidal and spheroidal voids. IEEE Transactions on Electrical Insulation, 24(2), 335-342. https://doi.org/10.1109/14.90292

\section{General rights}

Copyright and moral rights for the publications made accessible in the public portal are retained by the authors and/or other copyright owners and it is a condition of accessing publications that users recognise and abide by the legal requirements associated with these rights.

- Users may download and print one copy of any publication from the public portal for the purpose of private study or research.

- You may not further distribute the material or use it for any profit-making activity or commercial gain

- You may freely distribute the URL identifying the publication in the public portal

If you believe that this document breaches copyright please contact us providing details, and we will remove access to the work immediately and investigate your claim 


\title{
Partial Discharges in Ellipsoidal and Spheroidal Voids
}

\author{
G. C. Crichton, P. W. Karlsson \\ and $\mathrm{A}$. Pedersen \\ Physics Laboratory II, \\ The Technical University of Denmark, Lyngby, \\ Denmark.
}

\begin{abstract}
Transients associated with partial discharges in voids can be described in terms of the charges induced on the terminal electrodes of the system. The relationship between the induced charge and the properties which are usually measured is discussed. The method is illustrated by applying it to a spheroidal void located in a simple disk-type GIS spacer.
\end{abstract}

\section{INTRODUCTION}

$\mathrm{T}$ HE transients which are manifest at the electrodes of a system during partial-discharge activity are related to the charges which, in view of Faraday's ice-pail experiment, are induced on the electrodes. The sources of these induced charges are the charges which, as a result of this partial-discharge activity, are distributed within voids located throughout the system.

The induced charge can be expressed as the difference between the charge on the electrode when discharges have occurred, and the charge which would have been on the electrode had the system been discharge free [1]. The direct implementation of this approach could be rather cumbersome as it requires the solution of Poisson's equation.

A more straightforward approach is possible through an application of the principle of superposition $[2,3]$. This can be done in two ways depending on whether the analysis is based on the $P$-field [2] or on the $D$-field [3] in the dielectric. In practice, the application of the latter is more convenient, and this approach will therefore be employed in the present paper.
A discharge in a void results in a deployment of charges on the surface $S$ of the void. The surface-charge density $\sigma$ will attain such values that the field within the void will reduce until the discharge is quenched. In view of the principle of superposition, it is evident that the induced charge related to the charge distribution on $S$ can be expressed [3], in the form

$$
q=-\int_{S} \lambda \sigma d S
$$

in which $\lambda$ is a dimensionless scalar function which depends on the position of $d S$ only. The function $\lambda$ is given by Laplace's equation

$$
\vec{\nabla} \cdot(\varepsilon \vec{\nabla} \lambda)=0
$$

where $\varepsilon$ is the permittivity, [4]. The boundary conditions are $\lambda=1$ at the electrode on which $q$ is distributed, and $\lambda=0$ at all other electrodes. In addition, the following condition must be fulfilled at all dielectric interfaces

$$
\varepsilon_{+}\left(\frac{\partial \lambda}{\partial n}\right)_{+}=\varepsilon_{-}\left(\frac{\partial \lambda}{\partial n}\right)_{-}
$$

where $\lambda$ is differentiated in the direction normal to the interface and the signs + and - refer to the two sides

$0018-9367 / 89 / 0400-33581.00$ (C) 1989 IEEE 
of the interface. Since Equation (2) is Laplace's equation, any standard method for the calculation of spacecharge-free electrostatic fields can be used to evaluate $\lambda$. This is possible since the potential $V$ at a point can be expressed as $V=\lambda U$, where $U$ is the voltage applied in the field calculation.

Viewed from the electrode on which the induced charge $q$ is distributed, the charges deposited on $S$ can be considered, to a first approximation, as an electric dipole configuration since the net charge within the void remains zero. The dipole moment $\mu$ of the charges deposited on $S$ is given by

$$
\vec{\mu}=\int_{S} \vec{r} \sigma d S
$$

where $\vec{r}$ is a radius vector which locates the position of the surface element $d S$. The induced charge which arises from this dipole is given by [3],

$$
q=-\vec{\mu} \cdot \vec{\nabla} \lambda
$$

Although voids in epoxy spacers are usually close to spherical in shape, it is of advantage to consider transients caused by discharges in voids of more general geometry. Formulae for the dipole moment of relevant charge distributions on the surfaces of ellipsoidal voids are given. The effects of void size, shape and location on the magnitude of the induced charge are then discussed with particular reference to spheroidal voids.

\section{TRANSIENTS RELATED TO INDUCED CHARGES}

\footnotetext{
ATHough the observable transients are inherently A related to the induced charges, the properties which primarily are measured are transients in the applied voltage and current pulses in the lead to the terminal electrode. The relationship between these properties and the induced charge can be found in the following manner. Just prior to the first discharge in the void the potential of the electrode is $U$ and the associated charge is $Q$. We compare this with the situation immediately after the discharge is quenched. The potential has now dropped to $U-\Delta U$ and the charge on the electrode has become $Q+\Delta Q$, where $\Delta Q$ is the charge transferred to the electrode from the external source. Green's reciprocal theorem [5] then yields
}

$$
(U-\Delta U) Q=U(Q+\Delta Q)+\int_{S} V \sigma d S
$$

where $V$ is the scalar potential at the surface element $d S$ for the discharge-free situation. Since $V=\lambda U$ in consequence of Equations (2) and (3), and $Q=C U$, where $C$ is the capacitance of the system, we obtain

$$
-\int_{S} \lambda \sigma d S=C \Delta U+\Delta Q
$$

or

$$
q=C \Delta U+\Delta Q
$$

If the impedance of the circuit is large for the current which is associated with the discharge, then $C \Delta U$ can be much larger than $\Delta Q$. The induced charge is then given approximately by

$$
q \approx C \Delta U
$$

It should be emphasized that the capacitance of the system is not affected by partial discharges $[2,3]$ and that, as a consequence, the transients cannot be related to a change in the capacitance. The proper concept of capacitance [6] implies that the field between the electrodes is Laplacian, and the field is not Laplacian if space charges are present. However, if the principle of superposition is utilized, the actual field can be considered to be the sum of a space-charge Poisson field and the original space-charge-free Laplacian field. It is the latter which determines the capacitance of the system.

\section{ELLIPSOIDAL VOIDS}

$\mathrm{W}$ E consider an ellipsoidal void, the dimensions of which are so small that the internal field may be considered to be effectively uniform. Since any direction of the field within the ellipsoid can be resolved into three orthogonal components, each parallel to an axis, it is sufficient to consider the case for which the field is parallel to one of these axes.

A partial discharge can develop when the field within the ellipsoid reaches the inception value $E_{i}$. Such a discharge will result in a deposition of charges on the surface of the ellipsoid and a reduction in the internal field. The discharge will be quenched when this field is reduced to the limiting value $E_{l}$, i.e. the field below which ionization growth is impossible. To simplify the 
analysis we assume that the field within the ellipsoid remains uniform, and that the entire volume of the ellipsoid is involved in the discharge. In this case, the dipole moment can be quantified readily. Even if these assumptions are not fulfilled in practice, the general conclusions which can be drawn from the analysis will remain valid.

The electrostatics of ellipsoids is discussed in several advanced textbooks, e.g. [7]. From the field expressions given therein it can be proved that the dipole moment of the charge distribution left on the surface of an ellipsoid, following the above assumptions, may be written in the form

$$
\vec{\mu}=\frac{8 \pi \varepsilon}{3 A}\left[\vec{E}_{0}-\left[1+\frac{a b c A\left(\varepsilon_{o}-\varepsilon\right)}{2 \varepsilon}\right] \vec{E}_{l}\right]
$$

where $a, b, c$ are the semi-axes of the ellipsoid. $E_{0}$ is the ambient field when the internal field is equal to the inception field $E_{i}$; i.e.. $E_{0}$ is the field in the idealized (i.e. void-free) system at a location corresponding to that of the ellipsoidal void. $E_{0}, E_{i}$. and $E_{l}$ are all assumed to be parallel to the $a$-axis. The parameter $A$ is given by the integtal

$$
A=\int_{0}^{\infty} \frac{d s}{\left(a^{2}+s\right)^{\frac{3}{2}}\left(b^{2}+s\right)^{\frac{1}{2}}\left(c^{2}+s\right)^{\frac{1}{2}}}
$$

where $s$ is a dummy variable. The relationship between $E_{0}$ and the inception field $E_{i}$ is given by

$$
\vec{E}_{0}=\left[1+\frac{a b c A\left(\varepsilon_{o}-\varepsilon\right)}{2 \varepsilon}\right] \vec{E}_{i}
$$

$\varepsilon$ is the permittivity of the ambient dielectric and $\varepsilon_{o}$ is the permittivity of the gas within the ellipsoid; normally $\varepsilon_{o}$ can be assumed to be the permittivity of free space.

If we introduce the dimensionless parameters

$$
K=\frac{2}{a b c A}
$$

and

$$
h=\left[1+\frac{a b c A\left(\varepsilon_{o}-\varepsilon\right)}{2 \varepsilon}\right]^{-1}
$$

we may rewrite the dipole moment as

$$
\vec{\mu}=\left(\frac{K}{h}\right) \Omega \varepsilon\left(\vec{E}_{i}-\vec{E}_{l}\right)
$$

where $\Omega$ equals the volume $(4 \pi / 3) a b c$ of the ellipsoid. The parameters $K$ and $h$ are related by

$$
h=\frac{K \varepsilon_{r}}{1+(K-1) \varepsilon_{r}}
$$

where $\varepsilon_{r}=\varepsilon / \varepsilon_{o}$ is the relative permittivity of the bulk dielectric.
The induced charge is found by combining Equations (5) and (15) to give

$$
q=-\left(\frac{K}{h}\right) \Omega \varepsilon\left(\vec{E}_{i}-\vec{E}_{l}\right) \cdot \vec{\nabla} \lambda
$$

Since $\lambda$ is proportional to the scalar potential for the space-charge-free field we have, with reference to Equations (12) and (14), that

$$
\vec{\nabla} \lambda=h \vec{\nabla} \lambda_{0} \quad 1 \leq h \leq \varepsilon_{r}
$$

where $\lambda_{0}$ is the solution to Laplace's equation, Equation (2), at the location of the ellipsoid for the idealized (void-free) system. The induced-charge thus becomes

$$
q=-K \Omega \varepsilon\left(\vec{E}_{i}-\vec{E}_{l}\right) \cdot \vec{\nabla} \lambda_{0}
$$

and as $\lambda$ is eliminated with the introduction of $\lambda_{n}$ the calculation of the induced charge is dramatically simplified. For practical geometries the evaluation of $\lambda_{0}$ (void-free) is trivial in comparison to that of $\lambda$ (voidpresent).

\section{SPHEROIDAL VOIDS}

W Hen $b=c$ the ellipsoid becomes a spheroid, and W $A$ and $K$ are then expressible in terms of ln, arctan, and simpler functions. Introducing $b / a=x$, the dimensionless parameter $K$ for an oblate spheroid, i.e. $x>1$, is given by

$$
K=\frac{u^{3}}{\left(1+u^{2}\right)(u-\arctan u)}
$$

where $u=\sqrt{x^{2}-1}$. Similarly for a prolate spheroid, i.e. $x<1$, we have

$$
K=\frac{2 v^{3}}{\left(1-v^{2}\right)\left(\ln \frac{1+v}{1-v}-2 v\right)}
$$

where $v=\sqrt{1-x^{2}}$. In Figure $1, K$ is shown as a function of the axis ratio $a / b$.

The inception field $E_{i}$, depends on the pressure $p$ of the gas contained in the void and on the critical avalanche length $z_{0}[8]$. Since the field is parallel to the $a$-axis, the minimum value of $E_{i}$ will be associated with the maximum path length in the field direction, viz. 


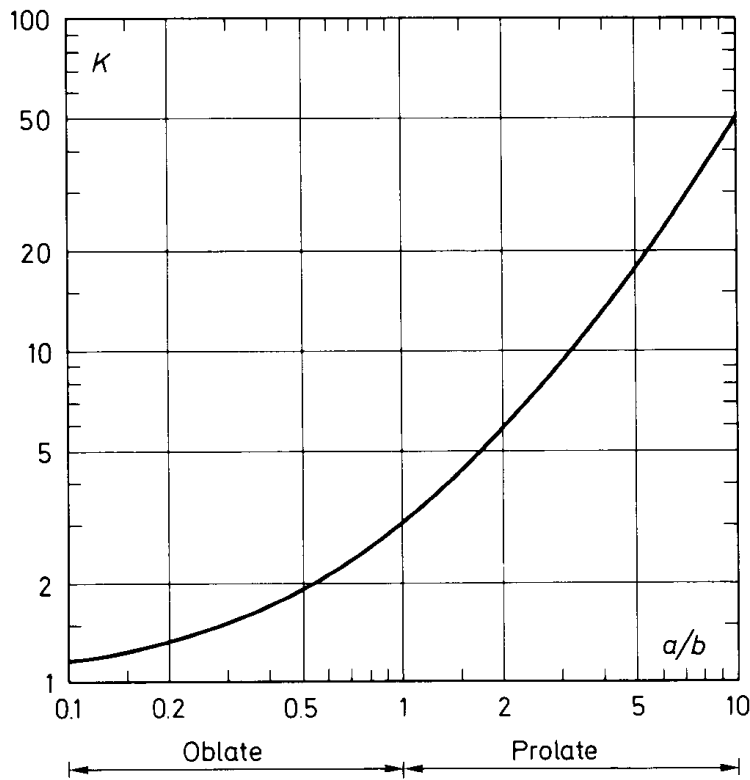

Figure 1 .

The parameter $K$ for spheroids. The applied field is parallel to the $a$-axis.

$z_{0}=2 a$. Consequently, for an electron attaching gas, the minimum value of $E_{i}$ will be given by

$$
\frac{E_{i}}{p}=\left[1+\frac{M}{2 a p}\right] \frac{E_{l}}{p}
$$

where $M$ is the figure of merit for the gas [8]. For $\mathrm{SF}_{6}$ ,$M=4 \mathrm{~Pa} \mathrm{~m}$ and $E_{l} / p=88.6 \mathrm{~V} / \mathrm{Pa} \mathrm{m}$. The dependence of $M$ upon $E_{l} / p$ is shown in Figure 2. The curve is valid for both unary gases and binary gas mixtures [9].

Similarly we have for non-attaching gases

$$
\frac{E_{i}}{p}=\left[1+\frac{B}{\sqrt{2 a p}}\right] \frac{E_{l}}{p}
$$

where $B$ is a constant which is characteristic for the gas [10]. For air $B=8.6(\mathrm{~Pa} \mathrm{~m})^{1 / 2}$ and $E_{l} / p=24.2 \mathrm{~V} / \mathrm{Pa}$ m.

The above $E_{i} / p$ expressions are derived via streamer breakdown criterion [8], and at the higher gas pressures these are seen to be identical in form to the Paschencurve breakdown functions [8]. In contrast, however, the above derivations do not invoke the presence of electrode boundaries. On this basis, the possible existence of a

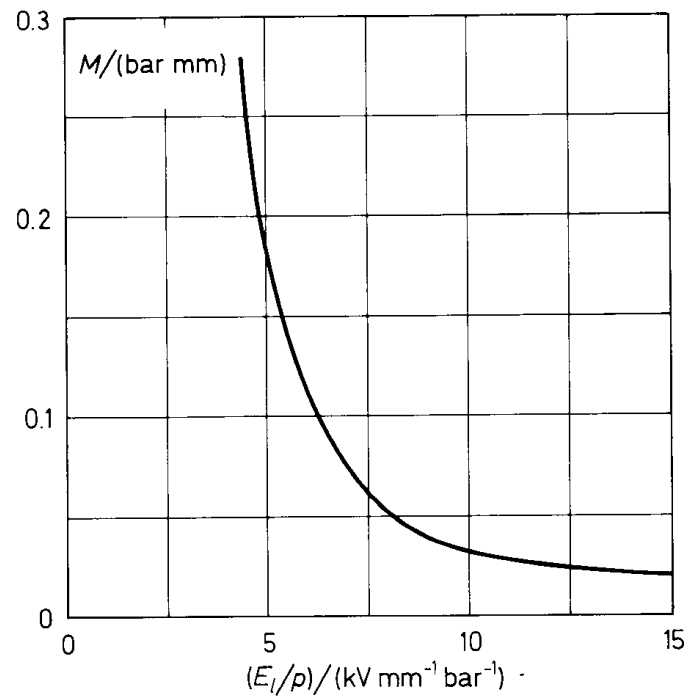

Figure 2.

The figure of merit $M$ as a function of $E_{l} / p$ for electronegative gases and gas mixtures.

minimum value for the onset voltage cannot be associated with the minimum voltage of the Paschen breakdown characteristic, as such a minimum is electrode dependent.

The streamer criterion, which depends on gas processes only, gives the minimum voltage level required to initiate a discharge; that is the onset level $U_{o n}$. An inherent assumption of this criterion is the existence of a suitably placed initiatory electron, such that the value of the statistical time-lag $t_{s}$ is zero. In general $t_{s}>0$, and hence if the applied voltage increases in time, the discharge will occur at a higher voltage level. This voltage is usually referred to as the discharge inception voltage $U_{i}$, with $U_{i}$ decreasing to $U_{o n}$ as $t_{s} \rightarrow 0$. In the present study $t_{s}$ is assumed to be zero. Thus inception and onset field strengths are synonymous, and $E_{i}$ is constrained to a minimum value.

From Equations (22) and (23) it is seen that

$$
E_{i}-E_{l}=\frac{M E_{l}}{2 a p}
$$

for an electron attaching gas, and

$$
E_{i}-E_{l}=\frac{B E_{l}}{\sqrt{2 a p}}
$$

for a non-attaching gas. It should be noted that for an attaching gas $\left(E_{i}-E_{l}\right)$ is independent of the pressure, since $E_{l}$ is proportional to pressure $p$. 
For a fixed location of the void in the system, $\vec{\nabla} \lambda_{0}$ will be constant. For constant gas pressure within the spheroid, we will study how the induced charge varies with the axial ratio $a / b$ for voids of constant volume. In this situation, the induced charge can be written in the form

$$
q=k_{\Omega} q_{1}
$$

where $q_{1}$ is the induced charge when $a / b=1$, i.e. for a spherical void, and $k_{\Omega}$ is a dimensionless shape-factor for constant void volume. Insertion of an expression for the semi-axis $a$ in terms of $\Omega$ and $a / b$ in Equations (19), (24) and (25) shows that, as $K=3$ for $a / b=1$, we have

$$
k_{\Omega}=\frac{K}{3}\left[\frac{a}{b}\right]^{-\frac{2}{3}}
$$

for an electron-attaching gas, and

$$
k_{\Omega}=\frac{K}{3}\left[\frac{a}{b}\right]^{-\frac{1}{3}}
$$

for a non-attaching gas. These shape-factors are thus independent of all the other properties of the gases which may be confined within the spheroid. The shape-factors for constant $\Omega$ are shown in Figure 3 as a function of $a / b$.

In addition to $k_{\Omega}$ it is of interest to consider the shape-factors $k_{a}$ and $k_{b}$ for constant $a$ and $b$, respectively. These shape factors are, for an attaching gas within the void, given by

$$
\begin{gathered}
k_{a}=\frac{K}{3}\left[\frac{a}{b}\right]^{-2} \\
k_{b}=\frac{K}{3}
\end{gathered}
$$

and for a non-attaching gas by

$$
\begin{aligned}
& k_{a}=\frac{K}{3}\left[\frac{a}{b}\right]^{-2} \\
& k_{b}=\frac{K}{3}\left[\frac{a}{b}\right]^{\frac{1}{2}}
\end{aligned}
$$

These shape factors are shown in Figures 4 and 5 , respectively, as a function of the axis ratio $a / b$. It should be noted that the void gas pressure is held constant as in the $k_{\Omega}$-evaluation.

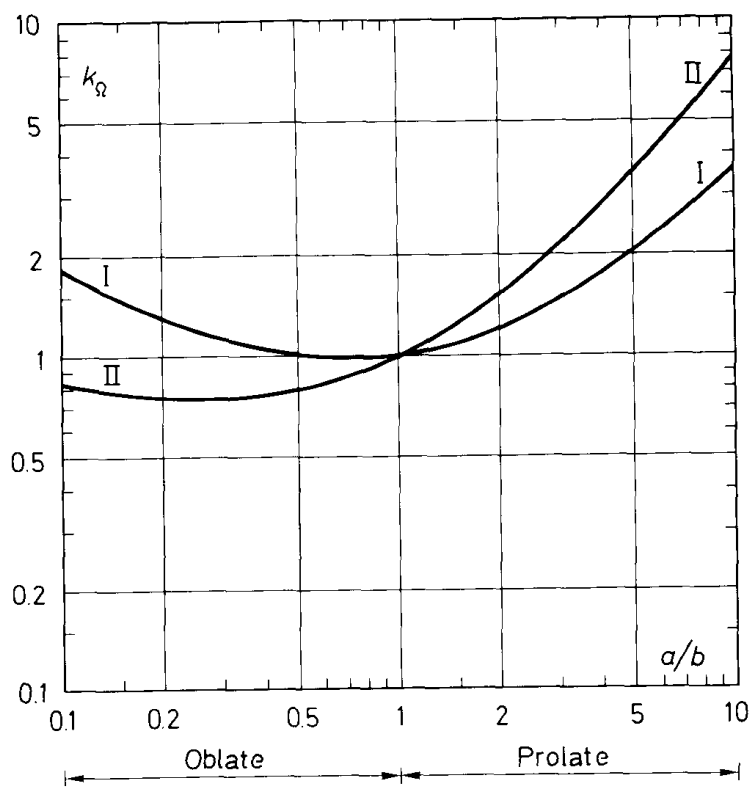

Figure 3.

The shape-factor $k_{\Omega}$ for spheroidal voids of constant volume. I: Attaching gases. II: Nonattaching gases.

\section{VOID IN A DISK-TYPE SPACER}

$\mathrm{W}$ E consider a coaxial electrode system with a simple disk-type spacer of relative dielectric permittivity $\varepsilon_{r}=4$. The radius of the inner electrode is $r_{1}=70 \mathrm{~mm}$, and the inner radius of the outer electrode is $r_{2}=190 \mathrm{~mm}$. Within the spacer is a spheroidal void of volume $\Omega=1 \mathrm{~mm}^{3}$. The center of the spheroid is located at $r=100 \mathrm{~mm}$ from the axis of the coaxial system. In the void is either $\mathrm{SF}_{6}$ or air at a pressure of $10^{5} \mathrm{~Pa}$.

For this simple geometry

$$
\vec{\nabla} \lambda_{0}=-\frac{\vec{e}_{r}}{r \ln \left(\frac{r_{2}}{r_{1}}\right)}
$$

where $\vec{e}_{r}$ is a unit vector perpendicular to the axis of the coaxial system and directed away from the inner electrode. Insertion of these data in Equations (19), (24)(28) and (33) gives the induced charge as a function of $a / b$. The results are shown in Figure 6 . The assumption that $t_{s}=0$ implies that the computed charge values are minimum values. 


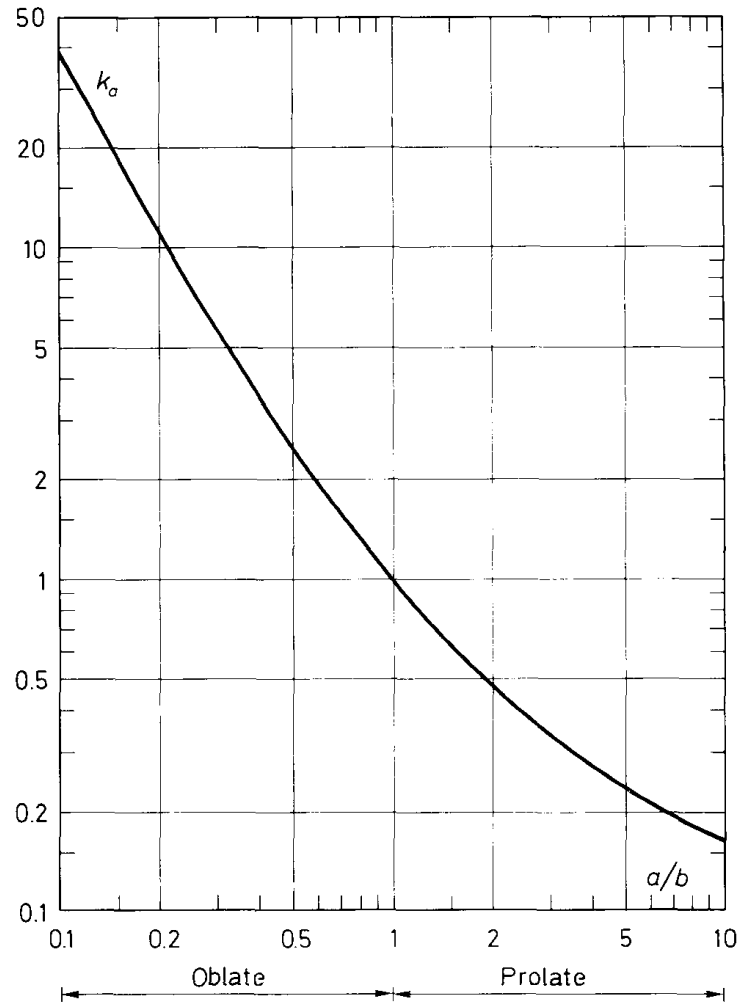

Figure 4.

The shape-factor $k_{\mathrm{a}}$ for spheroidal voids with constant length of the a-axis. Attaching and non-attaching gases.

The corresponding discharge inception voltages can be calculated from data obtained from Equations (22) and (23), and these voltages are shown in Figure 7. Although the inception voltage for $\mathrm{SF}_{6}$ is greater than that for air, it is seen that the induced charge is much smaller for the $\mathrm{SF}_{6}$-filled void than for the air-filled void. The reason is that, for equal $2 a p$-values, the difference $\left(E_{i}-E_{l}\right)$ is in general much smaller for $\mathrm{SF}_{6}$ than for air. Again, $t_{s}=0$ implies minimum inception voltage levels.

The shape-factors $k_{a}$ and $k_{b}$, which are distinctly different from $k_{\Omega}$ in their variation with $a / b$, may be employed in the calculations in a manner identical to the above. It is interesting to note that $k_{a}$ is independent of the void-gas in question, see Equations (29) and (31), and that in the range $0.1<a / b<10$ both $k_{a}$ and $k_{b}$ vary monotonically over approximately two orders of magnitude, with the former decreasing for increasing $a / b$.

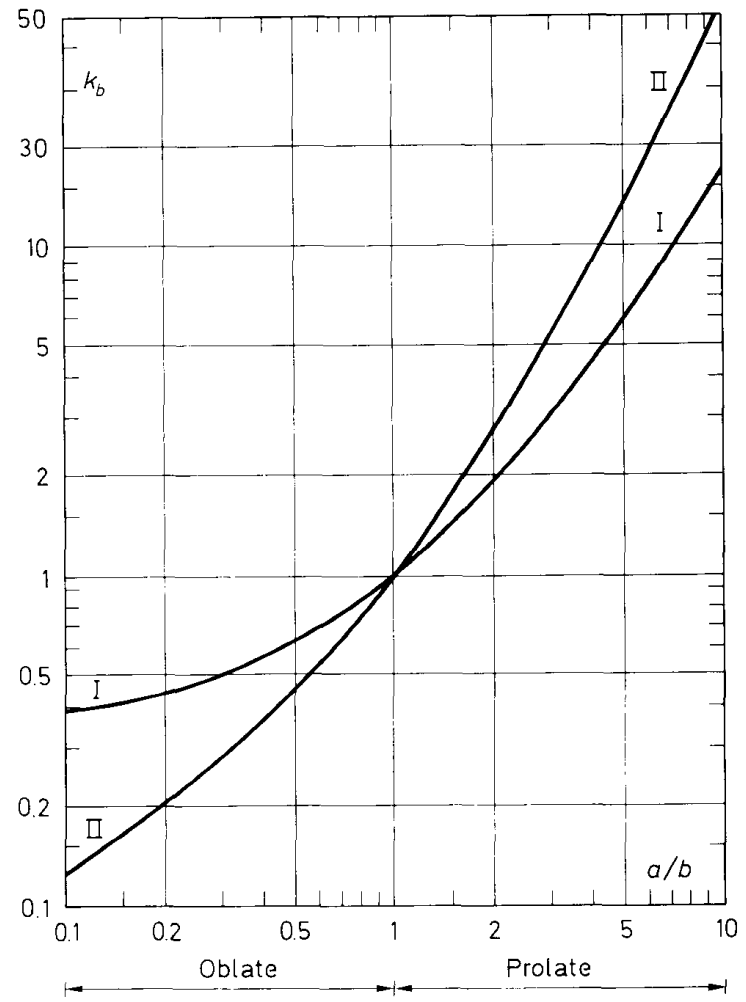

Figure 5.

The shape-factor $k_{b}$ for spheroidal voids with constant length of the $b$-axis. I: Attaching gases. II: Non-attaching gases.

\section{DISCUSSION}

\section{INDUCED-CHARGE CONCEPT}

T $\mathrm{HE}$ classical philosophy concerning the transients 1 which are related to partial-discharge activity is based on the assumption that the capacitance of the system is affected by the space charges which result from this discharge activity. This is, however, at variance with the concept of capacitance. The key to the electrodynamics of partial discharges is the concept of induced charge. Based on this concept, analytical expressions can be derived for the charges induced on the terminal electrode of a system. In the present study, the induced-charge-response to partial-discharge activity in ellipsoidal voids is derived. 


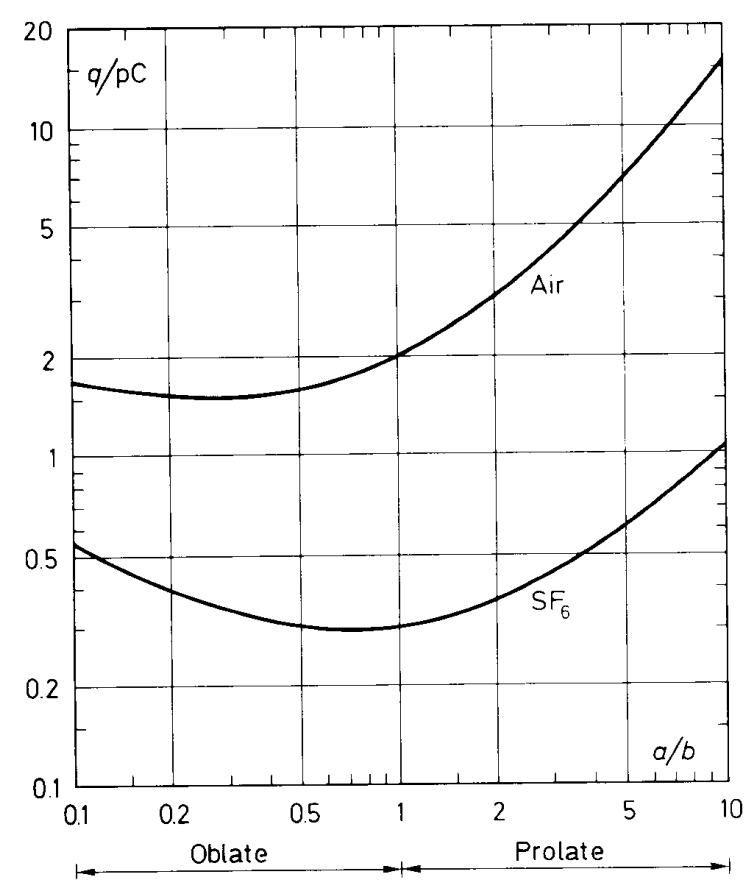

Figure 6.

Induced charge $q$ for spheroidal voids in a disktype spacer.

Location: $r=100 \mathrm{~mm}$ from the system axis. Volume: $\Omega=1 \mathrm{~mm}^{3}$. Electrode dimensions: $r_{1}=70 \mathrm{~mm}, r_{2}=190 \mathrm{~mm}$. Relative permittivity of dielectric: $\varepsilon_{r}=4$. Pressure within the void: $p=10^{5} \mathrm{~Pa}$.

\section{INFLUENCE OF VOID PARAMETERS}

The application of the concept to an actual insulating system is illustrated by considering a spheroidal void in a simple disk-type spacer. From the formulae obtained, conclusions can be drawn about the effects of the gas within the void on the induced-charge signal, together with the the effects of size, shape and void location.

For the specific case examined, i.e. $p=10^{5} \mathrm{~Pa}$, the non-attaching gas generates an induced charge which is approximately an order of magnitude larger than that generated by the attaching gas. In the latter case, however, inception voltages are higher by factors in the range 1.5 to 3 .

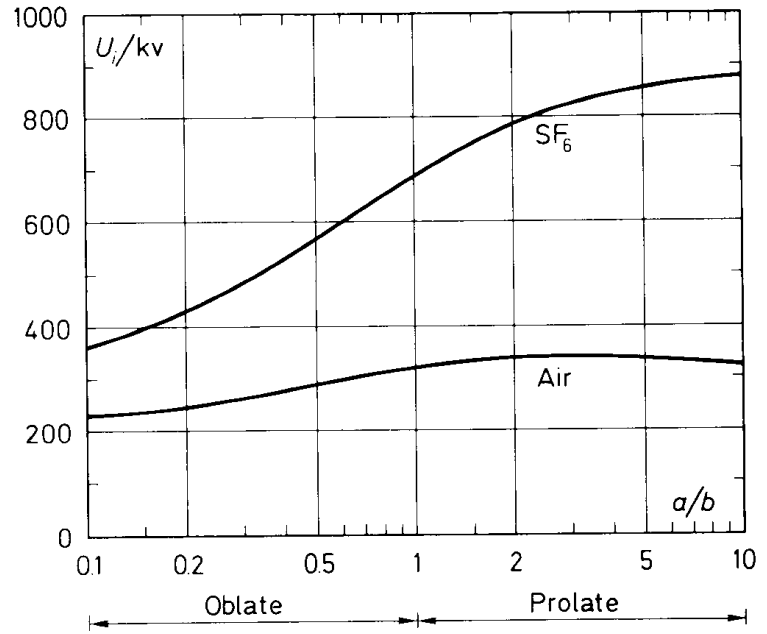

Figure 7.

Discharge inception voltages $U_{i}$ for the voids referred to in Figure 6.

\section{THE FUNCTION $\vec{\nabla} \lambda_{0}$}

The variation in induced-charge with location is given by the function $\vec{\nabla} \lambda_{0}$, and its introduction greatly simplifies the calculation of induced charge. For practical systems, which are always associated with nonuniform fields, the calculation of $\lambda_{0}$ (void-free) is essentially a trivial problem in comparison to the evaluation of $\lambda$ (void-present).

The function $\vec{\nabla} \lambda_{0}$ depends on location in the same manner as the field-strength in the Laplacian electrostatic field of the idealized (void-free) system. For a simple disk-type spacer the induced-charge is therefore proportional to the inverse of the distance from the axis of the electrode system to the center of the void.

\section{LIMITATIONS}

For the void geometries considered, it is more probable that the actual partial-discharge activity would occur in the vicinity of the void axis. However, by considering the entire volume of the void to be active in the discharge process, quantitative values can be readily ascribed to the induced charge characteristics. This is not possible if only an axial discharge location is considered, as in this latter case the dipole moment would remain obscure. The values of induced charge derived 
in the present analysis should therefore be interpreted as upper limiting values in the case $t_{s}=0$.

\section{PRACTICAL ASPECTS}

Finally, Equations (1) and (5) imply that the same value of induced charge could be associated with an infinite number of charge patterns and locations. Consequently, an exact knowledge of void location and geometry, gas pressure and composition will be required if a unique interpretation of the induced-charge signal and associated inception-voltage level is to be achieved. In practice, however, these restrictions should not prohibit a sound qualitative evaluation of the system insulation to be made on the basis of such measurements.

\section{CONCLUSION}

$\mathrm{T}$ HE correct explanation of partial-discharge transients can be attained only through the concept of induced charge. The application of this concept has enabled a partial-discharge theory to be developed through which the influence of all relevant void parameters can be correctly assessed. In contrast, the widely adopted $a b c$-capacitance model [11] does not allow this insight to be achieved. In addition, the abc-model is based on an erroneous application of the concept of capacitance, and, although this simple approach can be a useful tool when discussing measuring techniques, it may lead to quite incorrect conclusions if quantitative assessments are attempted.

\section{ACKNOWLEDGEMENT}

$\mathrm{T}$ HE authors wish to thank the Electric Power Research Institute for supporting this work through project RP2669-1.

\section{REFERENCES}

[1] H. Repp, K. W. Nissen and P. Röhl, "Partial Discharges in Voids: Inception Conditions and Detection Limits", Siemens Forsch.- u. Entwickl.-Ber., Vol. 12, pp. 101-106, 1983.
[2] A. Pedersen, "Current Pulses Generated by Discharges in Voids in Solid Dielectrics. A Field Theoretical Approach", Conference Record of the 1986 IEEE International Symposium on Electrical Insulation, IEEE Publication 86 CH2196-4-DEI, pp. 112-114, 1986.

[3] A. Pedersen, "Partial Discharges in Voids in Solid Dielectrics. An Alternative Approach", 1987 Annual Report - Conference on Electrical Insulation and Dielectric Phenomena, IEEE Publication $87 \mathrm{CH} 2462-$ 0, pp. 58-64, 1987.

[4] A. Pedersen, "On the Electrostatics of Probe Measurements of Surface Charge Densities", L. G. Christophorou and D. W. Bouldin (Eds.), Gaseous Dielectrics V, Pergamon Press NY, pp. 235-240, 1987.

[5] P. C. Clemmow, An Introduction to Electromagnetic Theory, Cambridge University Press Cambridge, 1973 .

[6] J. Fischer, Elektrodynamik, Springer-Verlag Berlin, 1976.

[7] J. A. Stratton, Electromagnetic Theory, McGraw-Hill New York, 1941.

[8] A. Pedersen, I. W. McAllister, G. C. Crichton and S. Vibholm, "Formulation of the Streamer Breakdown Criterion and its Application to Strongly Electronegative Gases and Gas Mixtures", Archiv für Elektrotechnik, Vol. 67, pp. 395-402, 1984.

[9] J. Berril, J. M. Christensen and I. W. McAllister, "Measurement of the Figure of Merit $M$ for $\mathrm{CBrClF}_{2}, \mathrm{CBr}_{2} \mathrm{~F}_{2}, \mathrm{CBrF}_{3}$ and $\mathrm{CClF}_{3}$ ", 5th International Symposium on High Voltage Engineering, Braunschweig 1987. Vol. 1, Paper no.15.16.

[10] 1. W. McAllister and A. Pedersen, "Corona-Onset Field-Strength Calculations and the Equivalent Radius Concept", Archiv für Elektrotechnik, Vol. 64, pp. 43-48, 1981.

[11] S. Whitehead, Dielectric Breakdown of Solids, Clarendon Press Oxford, 1951.

Manuscript was received on 21 Feb 1989 